\title{
Specific tracheal migration is mediated by complementary expression of cell surface proteins
}

\author{
Muriel Boube, ${ }^{1,4}$ Maria D. Martin-Bermudo, ${ }^{2}$ Nicholas H. Brown, ${ }^{2,3}$ and Jordi Casanova ${ }^{1,5}$ \\ ${ }^{1}$ Institut de Biologia de Biologia Molecular de Barcelona (CSIC), 08034 Barcelona, Spain; ${ }^{2}$ Department of Anatomy, \\ Cambridge University, Cambridge CB2 3DY, UK; ${ }^{3}$ Wellcome/Cancer Research Campaign, Cambridge CB2 1QR, UK
}

\begin{abstract}
Migration of the Drosophila tracheal cells relies on cues provided by nearby cells; however, little is known about how these signals specify a migratory path. Here we investigate the role of cell surface proteins in the definition of such a pathway. We have found that the PS1 integrin is required in the tracheal cells of the visceral branch, whereas the PS2 integrin is required in the visceral mesoderm; both integrins are necessary for the spreading of the visceral branch over its substratum. This is the first identification of a cell surface molecule with expression restricted to a subset of tracheal cells that all migrate in a given direction. We have also found that expression of PS1 in the visceral branch is regulated by the genes that direct tracheal cell migration, showing that integrin expression is part of the cell-fate program that they specify. These results support a model in which signal transduction determines the tracheal migratory pathways by regulating the expression of cell surface proteins, which in turn interact with surface molecules on the surrounding cell population.
\end{abstract}

[Key Words: Cell migration; integrins; Drosophila; tracheal system; morphogenesis]

Received December 6, 2000; revised version accepted April 24, 2001.

Cell migration is a widespread phenomenon in many biological processes, both in development and in the adult organism. Migrating cells extend processes that adhere to substrates and these adhesions are required to pull the cell; these have to be subsequently released to permit cell locomotion. In addition, cells move in an ordered pattern, following particular paths of migration, probably by recognition of distinct cues and substrates. Thus, the establishment of specific interactions between cells and their substrates is a crucial step in migration, a process ultimately determined by molecules expressed at their surface.

The tracheal system of Drosophila is a particularly tractable model for the study of cell migration, and, in particular, of the mechanisms that guide cells to migrate in specific directions (for review, see Hogan and Yingling 1998; Metzger and Krasnow 1999). The larval tracheal system of Drosophila is a complex tubular network that conducts oxygen from the exterior to the internal tissues. It arises from the tracheal placodes, clusters of ectodermal cells that appear at each side of 10 embryonic segments, from the second thoracic segment to the eighth abdominal segment. The cells of each cluster in-

\footnotetext{
${ }^{4}$ Present address: Centre de Biologie du Developpement, 31062 Toulouse Cedex, France.

${ }^{5}$ Corresponding author.

E-MAIL jcrbmc@cid.csic.es; FAX 34-93-2045904.

Article and publication are at http://www.genesdev.org/cgi/doi/10.1101/ $\operatorname{gad} .195501$.
}

vaginate and migrate in different and stereotyped directions to form each of the primary tracheal branches (Manning and Krasnow 1993). The general conclusion from many studies is that the direction of migration of the tracheal cells relies on a set of positional cues provided by nearby cells. On the one hand, branchless (bnl), a gene encoding an FGF homolog, is expressed around the developing tracheal system in clusters of cells at each position in which a new branch will form and grow. Activation of the Breathless (Btl) receptor in the tracheal cells by Bnl is thought to stimulate and guide tracheal migration toward these positions (Sutherland et al. 1996). On the other hand, Dpp, EGF, and Wnt signaling have a role in the choice between the alternative directions of migration (Llimargas and Casanova 1997; Vincent et al. 1997; Wappner et al. 1997; Chihara and Hayashi 2000; Llimargas 2000). However, it is not known how the signals from Dpp, EGF, and Wnt specify a particular migratory path or what cell surface proteins are used by the tracheal cells to interact with their specific substrates. In this work we have investigated the role of cell adhesion receptors of the integrin family in the definition of a migratory pathway.

\section{Results}

\section{Migration of the tracheal visceral branches}

We have focused our study on the tracheal cells that migrate over the visceral mesoderm to form the visceral 
branches, the ramifications of the tracheal tree that transport oxygen to the gut. They arise as small bulges in some of the centrally located pairs of tracheal pits (TP). In particular, visceral branches arise from six pairs of tracheal pits, TP2 and TP4 to TP8 (for a review of visceral branch formation, see Manning and Krasnow 1993). A distinctive feature of these branches is their direction of migration; whereas formation of the other primary branches is guided by $b n l$ expression in small clusters of epidermal cells, the outgrowth of the visceral branch is directed toward clusters of bnl-expressing cells in the mesoderm (Sutherland et al. 1996). At stage 11, the tracheal pits are located just over the clusters of the fat body precursor cells that are organized in metameric primordia (Franch-Marro and Casanova 2000). It is between these discrete regions of fat body precursors that the cells of the visceral branch contact the cells of the visceral mesoderm (Fig. 1A-D). Then, the cells of most visceral branches migrate anteriorly along the visceral meso-
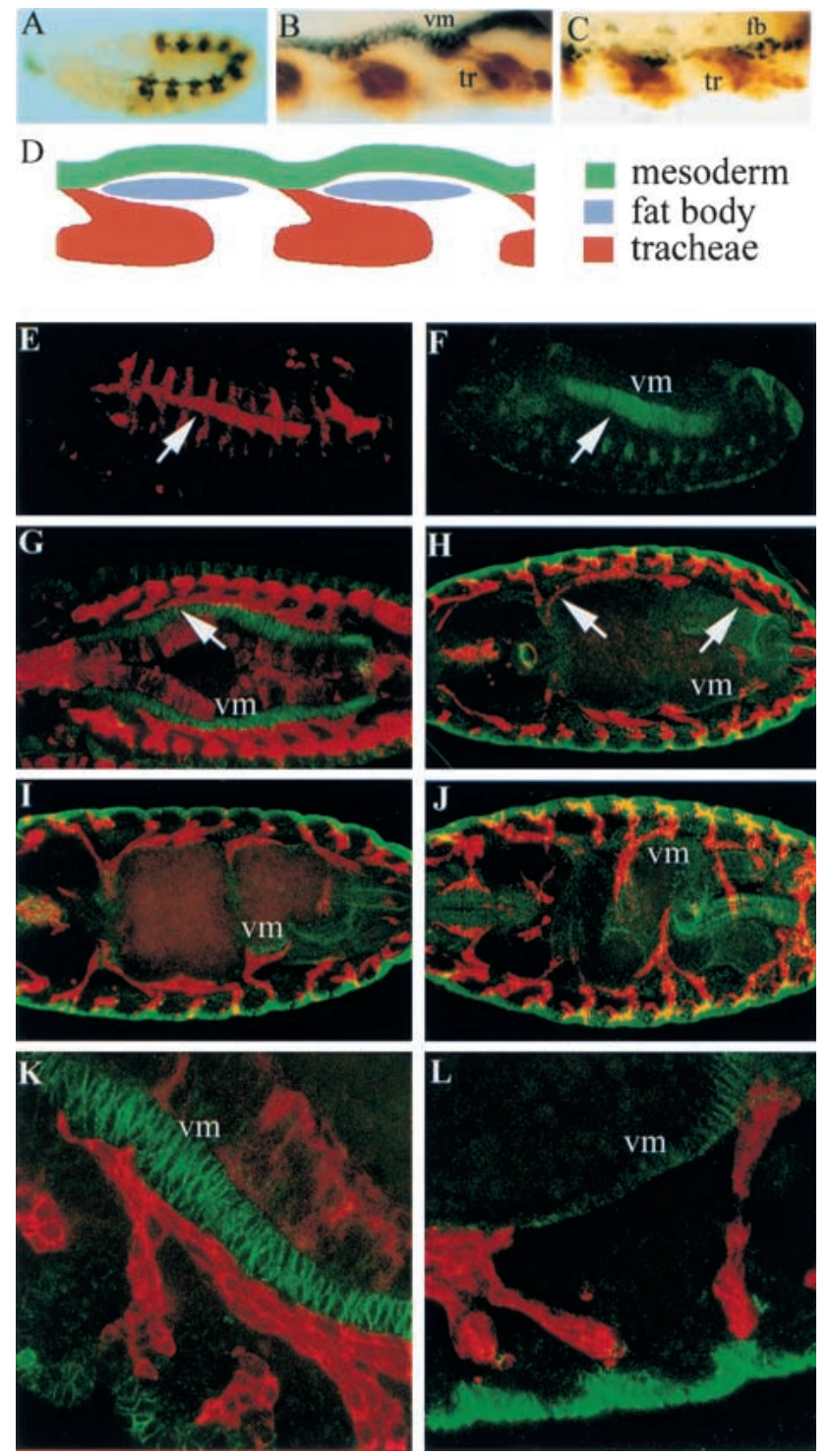

derm, with the exception of some cells of the branch derived from TP8, which migrate posteriorly (Fig. 1E-H). As development of the gut progresses, the visceral branches remain in contact with the visceral mesoderm and follow the convolutions that occur during formation of gut constrictions (Fig. 1I,J). Subsequently, fine branches form at positions in which visceral branches contact the gut.

\section{Specific expression of $\alpha$ PS1 in the tracheal cells of the visceral branches}

Only the tracheal cells that will form the visceral branch establish a distinct association with the visceral mesoderm. A mechanism to explain such a specific association could involve particular cell surface proteins being expressed in a complementary way in both the visceral mesoderm substrate and the migrating tracheal cells. We have described previously a similar scenario in which the visceral mesoderm serves as a substrate for the migrating primordial midgut cells. In this case, the migration requires a combination of PS1 and PS3 integrins in the migrating cells, and the PS2 integrin in the visceral mesoderm substratum (Martin-Bermudo et al. 1999). Integrins are a class of cell surface molecules formed as heterodimers of two single-pass type I transmembrane proteins, an $\alpha$ and a $\beta$ subunit (Hynes 1992; Cheresh and Mecham 1994). The Drosophila position-specific (PS) integrins are most similar to the $\beta 1$ family of vertebrate integrins, and one $\beta$ PS subunit is likely to form heterodimers with all five of the $\alpha$ PS subunits encoded by

Figure 1. Migration of the tracheal visceral branches. $(A)$ The tracheal pits in an embryo at stage 11 . Tracheal cells are visualized with an anti- $\beta$-galactosidase antibody in embryos from the 1-eve-1 enhancer trap line and the visceral mesoderm is detected by an anti-FasIII antibody. ( $B)$ Detail of an embryo at the same stage showing the tracheal cells (tr; in brown) and the visceral mesoderm (vm; in black). Note the cells of the visceral branches reaching the visceral mesoderm. $(C)$ Detail of an embryo at the same stage showing the tracheal cells (in brown as above) and the nuclei of the fat body precursor cells (fb; in black; detected by an anti-Srp antibody). Note the visceral branches between the groups of fat body precursor cells. $(D)$ Schematic representation of the initial development of the visceral branches reaching the visceral mesoderm between the fat body precursors. $(E-L)$ Tracheal cells are visualized in red by staining embryos carrying btl-GAL4 and UAS-tau-lacZ constructs with an anti- $\beta$-galactosidase antibody. We used an anti-FasIII antibody (in green) to visualize the visceral mesoderm (vm). $(E, F)$ Lateral view of a stage 12 embryo. The tracheal visceral branches $(E)$ follow the underlying visceral mesoderm $(F)$. $(G-J)$ Dorsal views. From stage $13(G)$ to stage $16(J)$, the visceral branches migrate along the visceral mesoderm; they follow the newly formed gut constrictions $(I)$ and ramify, to finally encompass all of the intestinal tract $(J) .(K, L)$ Higher magnification of anterior visceral branches of a stage 13 embryo $(K)$ and posterior visceral branches of a stage 14 embryo $(L)$ showing the tight association between the tracheal cells and the visceral mesoderm. 
the Drosophila genome (Brown et al. 2000). As with the majority of integrins, the PS integrins are thought to function by binding ligands within the extracellular matrix. To see whether integrins might be playing a role in tracheal migration, we examined their expression in the tracheal cells. We have found that around stage 11, the $\alpha \mathrm{PS} 1 \mathrm{mRNA}$ is specifically expressed in those tracheal cells that give rise to the visceral branch, although we also detect weak expression in the dorsal cells (Fig. 2BD). Localized expression in the visceral branch is more clearly evident at stage 12 , during germ-band retraction, when the main branches appear as protrusions (Fig 2A,EG). $\alpha$ PS1 mRNA expression is maintained during stage 13 as the visceral branch grows, and is lost by stage 14 (Fig. 2H-J). We have also analyzed whether there is expression of the $\alpha$ PS2 subunit in the tracheal cells, and did not detect expression (data not shown).

\section{Complementary integrin requirement in the tracheal cells of the visceral branches and in their migratory substrate}

The specific expression of the $\alpha$ PS1 subunit prompted us to analyze its role in the development of the visceral branch. The $\alpha$ PS1 subunit is encoded by the multiple edematous wings (mew) gene (Brower et al. 1995). We have found that in mew mutant embryos the visceral branches fail to develop properly (Fig. 3D). We observe that although the visceral branches migrate normally toward the gut and reach and contact the visceral mesoderm (Fig. 3E), they do not migrate normally along the visceral mesoderm (Figs. 3F and 4A). At a stage when the cells of the visceral branches have migrated along the visceral mesoderm in wild-type embryos, in mew mutant embryos, the visceral branches cells remain at their initial point of contact with the visceral mesoderm (Fig. 3F). Some delayed migration can also be observed at later stages. Other aspects of visceral branch development are not affected in mew mutant embryos; for instance, the mispositioned visceral branches form fine branches later in development (Fig. 3D).

As the PS2 integrin is expressed in the visceral mesoderm, the substrate of visceral branch migration, we have addressed whether it is also required for visceral branch formation. The $\alpha$ PS2 subunit is encoded by the inflated (if) gene and we have found that in if mutant embryos, visceral branch formation is also impaired (Fig. $3 \mathrm{G})$. As is the case for mew mutant embryos, the visceral branches of if mutant embryos reach the visceral mesoderm, but fail to migrate over it (Figs. 3H-I and 4D). However, the defects are usually milder than in mew mutant embryos. We also observe that later branching is not affected (Fig. 3G). The defect in visceral branch formation in if mutant embryos is not due to a failure of $b n l$ expression, as it appears at the right position in the visceral mesoderm (data not shown).

As both $\alpha \mathrm{PS} 1$ and $\alpha \mathrm{PS} 2$ subunits form heterodimers with the $\beta$ PS subunit, mutations in the myospheroid (mys) gene encoding this subunit will also simultaneously eliminate the PS1 and PS2 integrins (Brower et

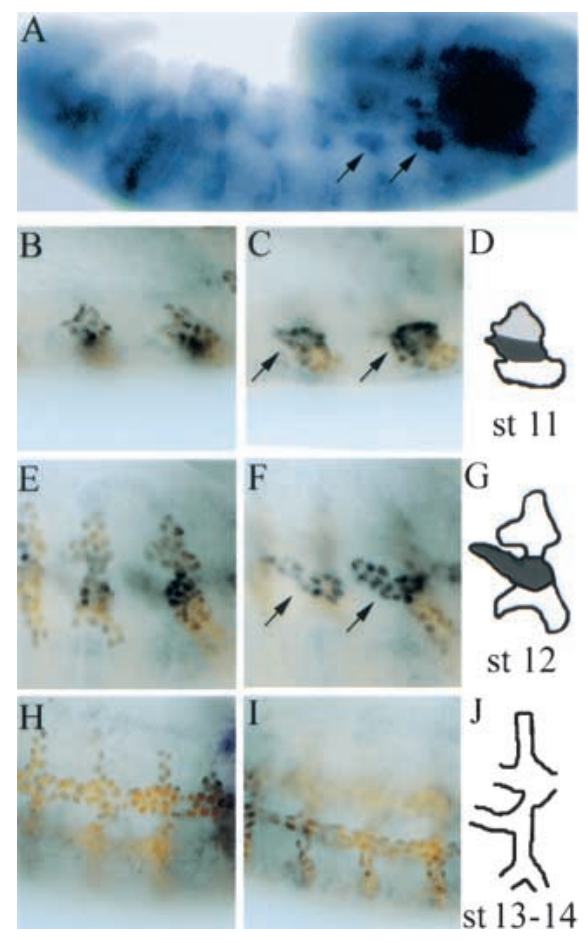

Figure 2. Expression of $\alpha \mathrm{PS} 1$ in the visceral branches. (A) $\alpha \mathrm{PS} 1$ expression in a stage 12 embryo visualized by in situ hybridization. Arrows point to the areas of $\alpha \mathrm{PS} 1$ expression that correspond to the cells of the visceral branch from TP4 and TP5. $(B-T)$ double-staining experiment to visualize both $\alpha \mathrm{PS} 1$ expression by in situ hybridization (blue), and the tracheal cells with an anti-Trh antibody (brown). Two focal planes are shown for each embryo, a more external view $(B, E, H)$ and a more internal view $(C, F, I)$ followed by a schematic diagram. $(B-D)$ At late stage 11 , $\alpha \mathrm{PS} 1 \mathrm{mRNA}$ is strongly expressed in the center of the tracheal placode, in the region that will give rise to the visceral branch. $(E-G)$ At stage 12, $\alpha$ PS1 expression is restricted to the cells of the visceral branch. $(H-J)$ This expression is transient because it is no longer detected by stage 14 . In the double-staining experiments, the $\alpha$ PS1 signal appears stronger in the nucleus because of the overlap with the nuclear signal from the anti-Trh antibody.

al. 1984; Wilcox et al. 1984; Leptin et al. 1989). The $\beta P S$ subunit is maternally deposited in the embryo, and, therefore, to examine the effect of complete loss of the $\beta P S$ subunit, we have generated embryos lacking both maternal and zygotic mys expression by making germline clones (referred to hereafter as mys mutant embryos) (see Material and Methods). All mys mutant embryos exhibit a failure in the migration of the cells of the visceral branches along the visceral mesoderm similar to those observed in the $\alpha \mathrm{PS}$ mutants (Fig. 3J-L). However, in mys mutant embryos, some visceral branches detach from the visceral mesoderm (Fig. 3L), suggestive of a more extensive failure in the establishment of cell-cell interactions than in mutants for individual $\alpha \mathrm{PS}$ subunits. Some mys $s^{-}$embryos also show gaps in the dorsal trunk, the more prominent branch of the tracheal tree, and defects in the dorsal branches (Fig. 3K). Thus, the 


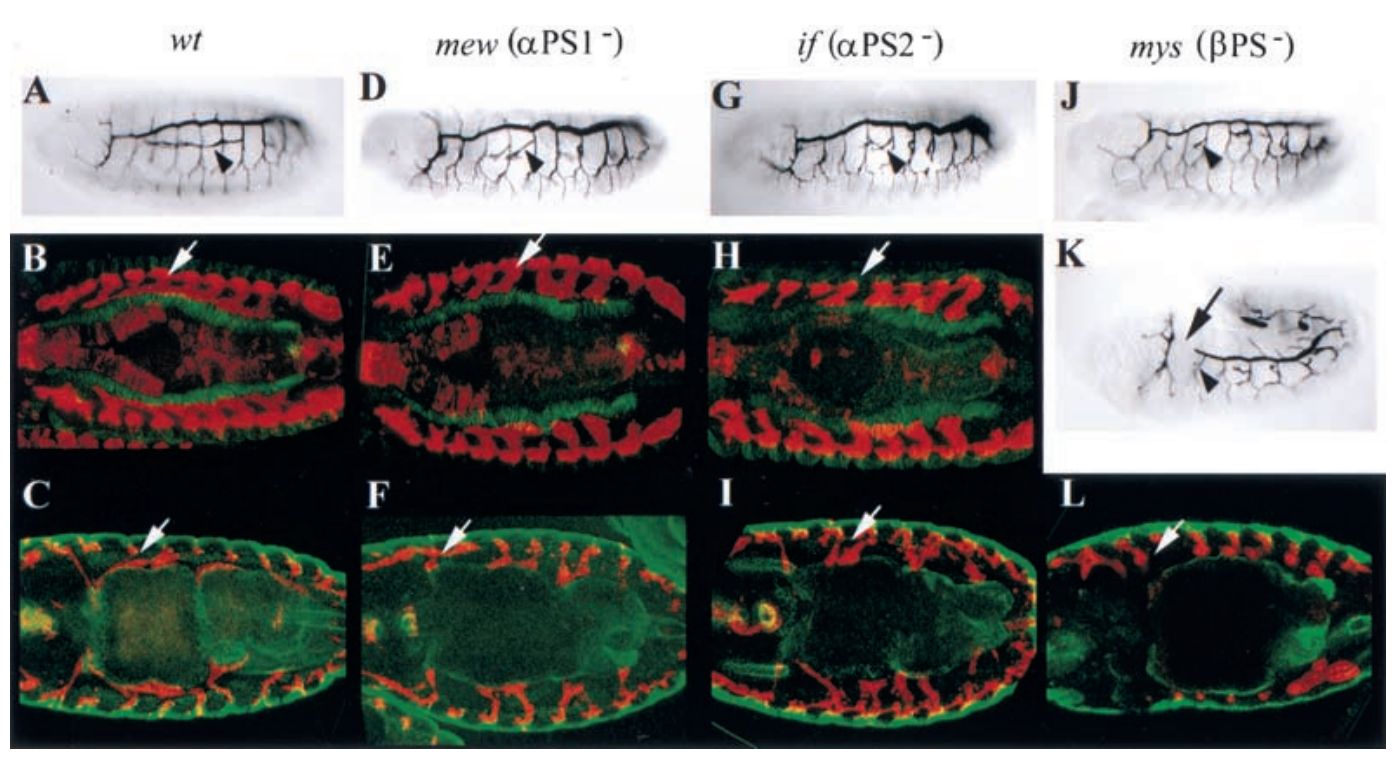

Figure 3. Migration of the visceral branches is impaired in integrin mutants. We examined the tracheal mutant phenotypes by using either the mAb2A12 antibody, which recognizes the tracheal lumen in stage 15 embryos $(A, D, G, J, K)$, or by using a combination of btlGAL4/UAS-tau-lacZ constructs and anti-FasIII antibody to analyze the relation between the tracheal cells and the mesoderm in stage $13(B, E, H)$ and stage 15 embryos $(C, F, I, L) .(A-C)$ Wild-type embryos. $(D)$ In mutant embryos for $\alpha$ PS1, the visceral branches (arrowhead) are shorter compared with the wild type, but they do have fine branches. Earlier in development, they reach and contact the visceral mesoderm $(E)$, but then they do not migrate along the mesoderm (arrow) $(F)$. $(G-I)$ The same defects on visceral branch migration are observed in mutants for $\alpha$ PS2, although they are often milder. $(J-L)$ Mutant embryos for $\beta P S$ also exhibit a failure in visceral branch migration similar to those observed in $\alpha$ PS mutants. $(L)$ In addition, visceral branches sometimes detach from the visceral mesoderm. $(K)$ Some mutant embryos for $\beta$ PS also show gaps in the dorsal trunk (arrow), and defects in the dorsal branch.

loss of all PS integrins produces stronger migration defects than the loss of $\alpha$ PS1 or $\alpha$ PS2 individually.

The above results suggest that PS1 ( $\alpha$ PS1 $\beta P S$ ) integrin is required in the migrating cells of the visceral branches, and PS2 ( $\alpha$ PS2 $\beta P S)$ integrin, in the visceral mesoderm substrate. If this is the case, we should be able to rescue the visceral branch phenotype of mutant embryos for each $\alpha$ subunit by expressing the appropriate subunit specifically in the cell population in which it is required. We find that targeted expression of $\alpha$ PS1 just in the tracheal cells can rescue the visceral branch phenotype of the mew $\left(\alpha \mathrm{PS}^{-}\right)$mutant embryos (Fig. $\left.4 \mathrm{~B}\right)$, although it causes some defects in the other branches (see below). Similarly, the visceral branch phenotype of if $\left(\alpha \mathrm{PS}^{-}\right)$ mutant embryos can be rescued by targeted expression of $\alpha$ PS2 just in the mesodermal cells (Fig. 4E). These results rule out the possibility that the defects in the visceral branches observed in integrin mutants are due to secondary consequences of disruption of other tissues. Furthermore, they also show that migration of the visceral branches requires the coordinated functions of PS1 integrin in the visceral branch and PS2 integrin in the visceral mesoderm (Fig. 4G).

Next, we tested whether the complementary expression of these two integrins is essential for tracheal migration. We analyzed the specific requirement of PS1 in the tracheal cells of the visceral branch by assessing whether the PS2 integrin could fulfill the role of PS1. We found that expression of $\alpha \mathrm{PS} 2$ in the tracheal cells does not rescue the defects in visceral branch migration caused by the lack of $\alpha$ PS1 in mew mutant embryos (Fig. 4C). This result indicates that PS1 function in particular, rather than PS integrin function in general, is required in the cells of the visceral branch for them to migrate along the visceral mesoderm. In contrast, we found that expression of $\alpha$ PS1 in place of $\alpha$ PS2 in the mesoderm does substantially rescue the visceral branch phenotype caused by lack of $\alpha$ PS2 (Fig. 4F). This finding suggests that either integrins can assemble a suitable substrate for PS1-dependent tracheal migration.

\section{Regulation of $\alpha$ PS1 in the cells of the visceral branches}

Previous clonal analysis has shown that cell lineage does not play an important role in the specification of the distinct identity of the different tracheal cells (Samakovlis et al. 1996). Instead, different signaling pathways act to spatially subdivide the tracheal placode by inducing groups of cells to migrate in a particular direction and acquire a specific morphology. This inductive process appears to be mediated by the differential activation of a set of transcription factors in discrete groups of tracheal cells. Therefore, we examined whether these transcription factors regulate the specific expression of the $\alpha \mathrm{PS} 1$ subunit.

The transcription factors knirps (kni) and knirps-related (knrl) are expressed in overlapping patterns during tracheal development in which they share redundant functions. They are both required in two phases of tracheal development. The initial tracheal expression of 


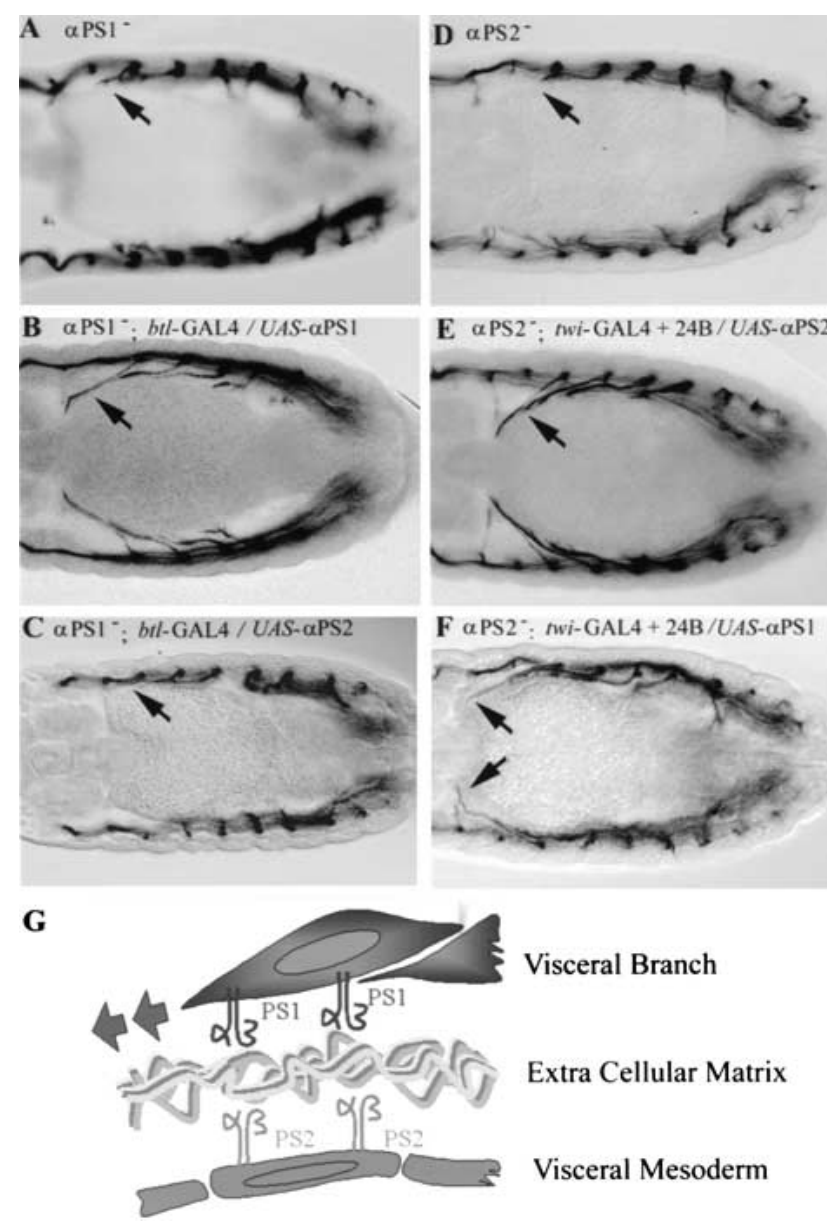

Figure 4. Tissue-specific requirement of $\alpha \mathrm{PS} 1$ and $\alpha \mathrm{PS} 2$. Dorsal view of tracheae at stage 14 embryos visualized by the mAb2A12 antibody. (A) A mutant embryo for $\alpha$ PS1. (B) Tracheal-specific expression of $\alpha$ PS1 using a $b t l-G A L 4$ line rescues the defects of visceral branch migration observed in mutant embryos for $\alpha$ PS1 (NB: these embryos also present defects in other tracheal branches; see Fig. 5). (C) Conversely, trachealspecific expression of $\alpha \mathrm{PS} 2$ does not rescue the visceral branch migration defect of $\alpha$ PS1 mutants. $(D)$ A mutant embryo for $\alpha$ PS2. (E) Mesoderm specific expression of $\alpha$ PS2 with the twi-GAL4 and the 24B lines rescues the defects of visceral branch migration observed in mutant embryos for $\alpha$ PS2. $(F)$ The visceral branch migration defect of $\alpha \mathrm{PS} 2$ mutants is also substantially rescued by specific expression of $\alpha \mathrm{PS} 1$ in the mesoderm. $(G)$ Diagram illustrating the requirement of $\alpha$ PS1 in the tracheal cells and $\alpha \mathrm{PS} 2$ is the mesoderm cells for visceral branch migration

kni/knrl appears around stage 10 and their activity is required early in the tracheal placode for primary branching outgrowth. Subsequently, kni/knrl expression becomes restricted to some branches, among them the visceral branches, and they are required for the directed outgrowth of these tracheal branches (Chen et al. 1998). As kni and knrl share redundant functions, we examined mutant embryos homozygous for a deficiency that uncovers both genes and whose effect on tracheal development is due solely to the lack of kni and knrl (Rothe et al.
1989|. In this mutant background, we found that $\alpha$ PS 1 is no longer expressed in the tracheal cells (Fig. 5B), indicating that these genes act as positive regulators of $\alpha$ PS1. To further assess the specific role of the late expression of $k n i / k n r l$ in the expression of $\alpha$ PS1 in the visceral branch, we have used a mutant combination that provides the segmentation and early tracheal kni expression, but not the later branch-specific expression of kni (see Material and Methods for the exact genotype). In these mutant embryos, there are also defects in visceral branch development and expression of $\alpha \mathrm{PS} 1$ is either absent or very much reduced (Fig. 5C). Thus, the late phase of kni/knrl branch-specific expression is specifically required for the proper expression of $\alpha$ PS1 in the cells of the visceral branches. However, kni/knrl are not sufficient to drive $\alpha$ PS1 expression, as they are expressed in other branches that do not express $\alpha$ PS1. Similarly, the $\alpha$ PS1 gene is not the only target of these transcription factors, as GAL4-driven expression of $\alpha$ PS1 is not able to rescue the phenotype of the deficiency (data not shown).

Another transcription factor that has an important role in specifying cell fate within the tracheal branches is encoded by spalt (sal). The expression of sal is restricted to the dorsal cells in the developing tracheal tree. By stage 12, sal is expressed only in the dorsal parts of the tracheal metameres, that is, in the cells of the dorsal trunk and the dorsal branches. By stages 14 and 15, sal expression declines in the cells of the dorsal branches, but remains high in the cells of the dorsal trunk (Kühnlein and Schuh 1996). We have found that in sal mutant embryos there is ectopic expression of $\alpha$ PS1 in the cells of the dorsal trunk and in the dorsal branches (Fig. 5D), indicating that sal activity is required to inhibit $\alpha \mathrm{PS} 1$ expression in the dorsal tracheal structures. In summary, these results indicate that $\alpha \mathrm{PS} 1$ expression is restricted to the cells of the visceral branches by the direct or indirect activities of the transcription factors that spatially subdivide the tracheal placode. The fact that $\alpha$ PS1 expression is regulated by the same genes that specify a particular cell fate, rather than independently, suggests that integrin expression is an important part of the cell fate decision.

\section{Control of integrin expression, migratory pathways, and morphogenesis}

To investigate the functional significance of the restricted expression of PS1, we examined whether it is important that the other tracheal branches do not express the PS1 integrin. We have induced the expression of $\alpha$ PS1 construct in all of the tracheal cells and found several defects in tracheal development, mainly in the dorsal branches. In wild-type embryos, each dorsal branch grows toward the dorsal midline, where it meets and fuses with the dorsal branch coming from the contralateral hemi segment. However, in embryos with ubiquitous tracheal $\alpha$ PS1 expression, many dorsal branches fail to fuse with their contralateral counterpart or fuse with an inappropriate branch (Fig. 5E). We also see occasional interruptions in the dorsal trunk of these 


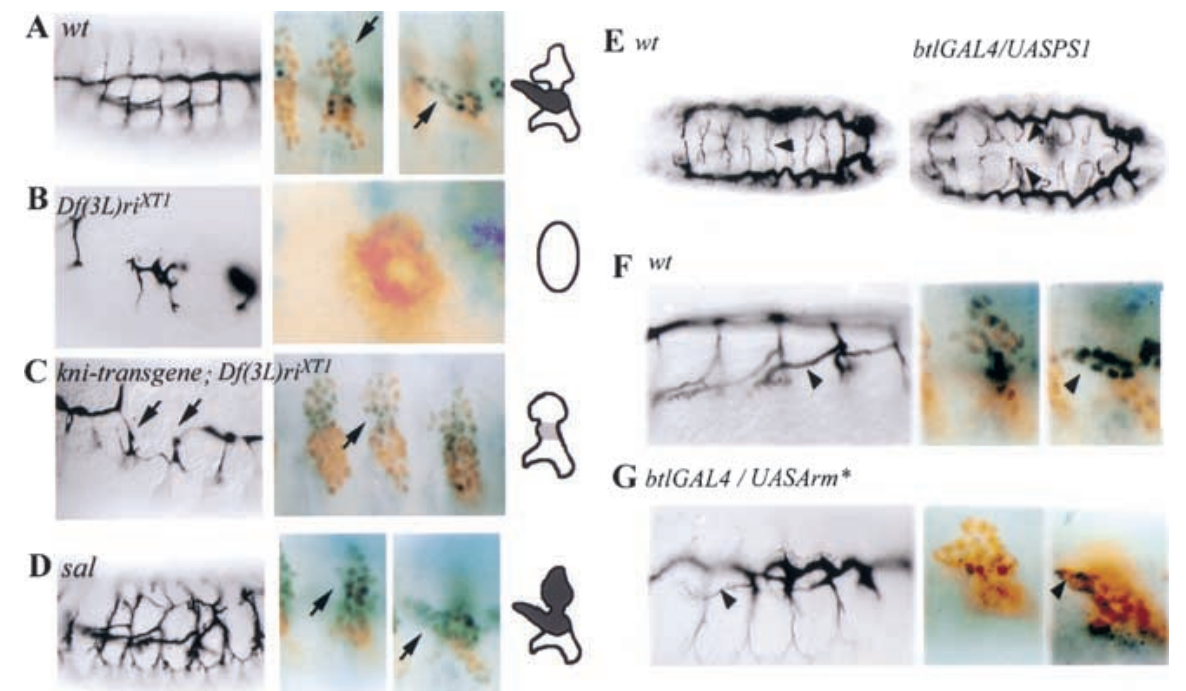

Tracheal migration and cell surface proteins

trunk; they usually lack the visceral branches and, if present, they are very rudimentary (arrows). In those embryos, $\alpha$ PS1 expression is absent or very much reduced, indicating that the branch-specific expression of kni/knrl is required for the proper expression of $\alpha \mathrm{PS} 1$ in the visceral branch. sal is specifically expressed in the dorsal part of the placode and is required for dorsal trunk formation $(D)$. In sal mutant embryos, we observe ectopic expression of $\alpha$ PS1 in the dorsal region of the tracheal metamere, indicating that sal is required to inhibit $\alpha$ PS1 expression in this region. (E) Dorsal view of stage 16 embryos. (Left) Wild-type embryo; (right) an embryo with expression of $\alpha$ PS1 in all the tracheal cells driven by btl-GAL4 and UAS- $\alpha$ PS1 constructs. In the wild-type embryo, the arrowhead points to the fusion between two dorsal branches from two contralateral hemisegments. In the $b t 1-G A L 4 / U A S-\alpha P S 1$ embryo, there is abnormal dorsal branch migration and fusion (arrowhead) and occasional dorsal trunk breaks (not shown). (F) Detail of a lateral view of a wild-type embryo at stage 16 (arrowhead points to a visceral branch) and two focal planes of a wild-type stage 12 embryo showing $\alpha$ PS1 expression in the cells of the visceral branch. $(G)$ Detail of a lateral view of an embryo carrying the UAS-Arm ${ }^{\star}$ (activated) and btl-GAL4 constructs; the visceral branches of these embryos are thinner and shorter (arrowhead) than in wild-type embryos. Two focal planes of a stage 12 embryo carrying the UAS-Arm* and btl-GAL4 constructs show that the number of cells expressing $\alpha$ PS1 is very much reduced. In $A-G$, the tracheal tree is visualized with the mAb2A12 antibody, the $\alpha$ PS1 expression is visualized by in situ hybridization (blue), and the tracheal cells are detected with an anti-Trh antibody (brown).

embryos (data not shown). However, we do not observe redirection of migration in this situation

The above results suggest that different groups of tracheal cells need to express different distinct adhesion molecules to migrate on the correct substrate. If this is the case, one would expect changes in the migratory behavior of these cells to be accompanied by changes in the expression of the adhesion molecules. A change in the path of migration of some tracheal cells has been observed in particular mutant combinations of the wingless $(w g)$ pathway. The $w g$ pathway is required for the formation of the dorsal trunk, and upon ubiquitous tracheal activation of the $\mathrm{wg}$ pathway, cells that would normally contribute to the visceral branch appear to adopt a dorsal trunk fate and change their path of migration accordingly (Chihara and Hayashi 2000; Llimargas 2000). As a result, the dorsal trunk becomes larger and fewer cells migrate along the visceral mesoderm. We find that in these mutant embryos, fewer cells express $\alpha$ PS1 (Fig. 5G). Thus, the signaling pathways that mediate the choice between alternative paths of migration regulate the appropriate expression of adhesion molecules in subsets of the tracheal cells.

\section{Discussion}

In this work, we have investigated how a group of cells select and recognize their migratory pathway in a model migratory process, the Drosophila tracheal system development. We have found that cell surface receptors of the integrin family mediate the migration of the visceral branch, one of the six primary tracheal branches. The PS1 integrin is required in the migrating cells of the visceral branch and the PS2 integrin is required in its substratum, the visceral mesoderm. This is the first identification of an adhesion molecule specifically expressed in a subset of tracheal cells that all migrate in a given direction. Furthermore, we have shown that $\alpha$ PS1 expression is restricted to the visceral branch by the activities of transcription factors situated downstream of the signaling pathways that spatially subdivide the tracheal placode. These results suggest a model by which signaling pathways mediate the choice between alternative migratory pathways by regulating the expression of cell surface proteins in subsets of tracheal cells.

\section{Specific requirements for the distinct integrin subunits}

Our rescue experiments show that migration of the visceral branches requires PS1 ( $\alpha$ PS1 $\beta$ PS) in the migrating cells of the visceral branches and PS2 ( $\alpha$ PS2 $\beta P S)$ in the visceral mesoderm substrate. However, they also show that whereas the PS2 integrin cannot fulfill the role of PS1 in the tracheal cells, PS1 can almost completely substitute for PS2 in the visceral mesoderm. This is consistent with the observation that the visceral branch de- 
fects in embryos lacking $\alpha$ PS2 are milder than in embryos that lack $\alpha \mathrm{PS} 1$, which already suggested a more strict requirement for integrin function in the migrating cells than in the substratum. Similarly, during midgut migration, loss of integrin function in the visceral mesoderm produces a much milder phenotype than the loss in the migrating midgut cells (Martin-Bermudo et al 1999|. The results presented here show that either $\alpha$ PS1 or $\alpha$ PS2 integrin on the surface of the visceral mesoderm is capable of producing a substrate containing a ligand that supports migration, but that only PS1 is capable of leading migration on that substrate. Interestingly, it should be noted that the visceral mesoderm functions as a two-sided migratory substratum: The endodermal cells of the midgut migrate along the internal side and the tracheal cells migrate along the external side. In both cases, their interaction involves the same proteins, PS2 in the substratum and PS1 in the migrating cells.

Our results also suggest a potential role of integrins in the migration of other tracheal branches. Removing the lone $\beta$ PS subunit, the obligatory partner for all $\alpha$ PS subunits, produces more extensive migration defects than the loss of $\alpha$ PS1 or $\alpha$ PS2. The lack of $\beta$ PS also affects dorsal trunk and dorsal branch migration in addition to the visceral branch. At least two alternative explanations for these additional defects can be proposed. On one hand, $\beta$ PS could be required in the dorsal branch and the dorsal trunk in combination with any of the other three alpha subunits, $\alpha$ PS3, $\alpha$ PS4, or $\alpha$ PS5. On the other hand, these mys mutant embryos may exhibit other phenotypes such as failure in germ-band retraction and dorsal closure. This variability of the phenotypes of embryos missing both the maternal and zygotic mys expression, described previously by Roote and Zusman (1995), leaves open the possibility that the defects observed in the dorsal branches and dorsal trunk are a secondary effect, due to alterations in other tissues.

\section{Integrins mediate the migration of the visceral branch} cells once they have reached their substrate

We have found that the localized $\alpha$ PS1 expression in the visceral branch depends on the transcription factors Kni, Knrl, and Sal that act to subdivide the tracheal placode. This spatial restriction is important because ectopic expression of $\alpha$ PS1 in all of the tracheal cells produces defects in dorsal trunk and dorsal branch migration. How- ever, redirection of the migration of these tracheal branches toward a different target is not observed. One possible explanation for these results is that steric hindrance by other tissues could impede these cells from adopting a new migratory pathway. Thus, the initial topology of the tracheal pits could also be critical in the assignment of a particular migratory pathway of each subset of tracheal cells. For example, dorsal tracheal cells may not be able to follow a visceral branch pathway if they cannot reach the visceral mesoderm, even under conditions of ectopic expression of $\alpha$ PS1. Another explanation, compatible with the previous one, is that multiple proteins could be required to define the particular migratory pathway of each subset of tracheal cells.

In this context, it should be noted that the initial migration of the visceral branches to the visceral mesoderm is not impaired in the integrin mutants. Therefore, it is possible that different molecules could be responsible for the migration of the tracheal cells of the visceral branch at different steps of their pathway. Alternatively, the initial migration toward the different clusters of bnl-expressing cells would be fixed by the topology of the tracheal pits; only the appropriate combination of cell surface molecules would then stabilize and allow the spreading of the different branches over their distinct substrates. In both hypotheses, integrins would only mediate the migration of the tracheal cells of the visceral branch after they have reached the visceral mesoderm.

\section{A general model for cell migration}

We have shown previously that the alternative migratory pathways of the tracheal cells are associated with distinct subsets of mesodermal cells (Franch-Marro and Casanova 2000). Here we provide the first identification of cell surface receptors that mediate these specific interactions. These results support a model in which signaling by transduction pathways specifies the particular migratory pathways of tracheal cells by regulating a precise array of adhesion proteins such as integrins at their surface (Fig. 6). This can be a very general mechanism to regulate integrin expression in distinct subpopulations of cells within a wider field. For instance, many genes operating in the morphogenesis of the insect tracheal system and in the vertebrate lung are functionally conserved despite the important differences that exist between the two systems (Metzer and Krasnow 1999). In
Figure 6. Schematic representation of the regulatory cascade that controls $\alpha$ PS1 expression in the tracheal cells of the visceral branch. Theses results suggest a more general model by which signaling by transduction pathways specifies the distinct cell migratory pathways by regulating the presence of specific proteins at their cell surface

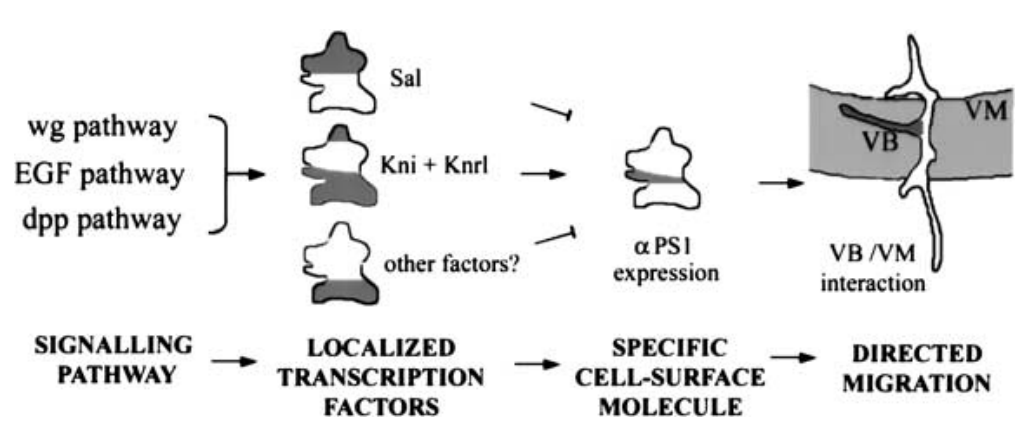


this scenario, it is worth noting that distinct integrin subunits are differentially expressed in the cells involved in mesenchymal/epithelial interactions during lung development (Wu and Santoro 1996). Thus, as in the case of the Drosophila tracheal system, control of cell surface molecules by signaling pathways in other systems may be involved in the mechanism conferring the specificity of cell-cell adhesions in migration and in other biological processes.

\section{Materials and methods}

Drosophila strains

The mutants used in this study are the null alleles if ${ }^{B 4}$, mew $^{m 6}$, mys $^{X G 43}$, and salII ${ }^{A 55}$. The deficiency $D f(3 L) r i^{X T 1}$ uncovers both the kni and knrl genes (Rothe et al. 1989); to rescue the segmentation and early tracheal kni expression, we used a kni transgene that rescues the kni segmentation phenotype and the kni primary branching phenotype in combination with the $D f(3 L) r i^{X T 1}$ (Chen et al. 1998). 1-eve-1 is an enhancer trap line specifically expressed in the tracheal system (Perrimon et al. 1991). To target gene expression to particular cells, we used the GAL4 system (Brand and Perrimon 1993). We used a combination of two GAL4 lines, twist-GAL4 and 24B, to drive gene expression in the mesoderm as described in Martin-Bermudo et al. (1997), and btl-GAL4 for expression in the tracheal cells (Shiga et al 1996). To overactivate the wg pathway, we have used a constitutively active form of armadillo (UAS-armS10; Pai et al. 1997). The UAS- $\alpha$ PS1 3.5 and UAS- $\alpha$ PS2 1.3 lines have been described previously in Martin-Bermudo et al. (1997). The UAS-tau-lacZ gene is described in Hidalgo et al (1995).

\section{Generation of germ-line clones}

We generated germ-line clones for $m y s^{X G 43}$ using the FLP-recombinase system and the dominant female sterile mutation ovo ${ }^{D 1}$ (Chou and Perrimon 1992). Virgin females of the genotype y m ys $^{X G 43} F R T^{101}$; UAS-tau-lacZ were crossed with $W$ OVO $^{D 1} F R T^{101}$; FLP $P^{F 38}$ males. The larvae of this cross were heat shocked three times at first, second, and third instar at $37^{\circ} \mathrm{C}$. The females $y \mathrm{~W}$ mys $^{X G 43} F R T^{101} / \mathrm{W}$ ovo ${ }^{D 1} F R T^{101}$; UAS-taulacZ/FLP $P^{F 38}$ from the cross were mated to FM7lacZ; bt1-GAL4 males to drive $\beta \mathrm{Gal}$ expression in the tracheal cells of the offspring. Only the embryos lacking both maternal and zygotic mys expression were analyzed.

\section{Antibody staining and in situ hybridization}

Embryos were staged according to Campos-Ortega and Hartenstein (1985), and stained according to standard protocols. We used the following primary antibodies: mouse 2A12 (DSHB, University of Iowa), which recognizes the lumen of the tracheal tree, rabbit anti-Srp (from R. Reuter, University of Tübingen, Germany), rat anti-Trh (from S. Crews, University of North Carolina, Chapel Hill), mouse DA1B5 anti-fasciclin III (from D. Brower, University of Arizona, Tuscon), and rabbit anti- $\beta$ Gal (Cappel). We used biotin-labeled secondary antibodies, followed by the vectastain Elite ABC kit (Vectorlabs) enhancement for standard experiments. For double labeling, we used $8 \% \mathrm{NiCl}_{2}$ in the first immunostaining to obtain dark color. For immunofluorescence, we used an anti-Rabbit secondary antibody coupled with Texas Red (Jackson Immuno Research Lab) to visualize tracheae, and a biotinylated anti-mouse antibody followed by streptavidin-fluorescein treatment (Jackson Immuno Research Lab) to label the mesoderm. Whole-mount in situ hybridization were done with a mew anti-sense RNA probe labeled with digoxygenin-UTP (Boerhringer-Mannheim). For antibody/in situ hybridization double-labeling experiments, we followed the procedure described in Manoukian and Krause (1992). Stained embryos were mounted and photographed either with a ZeissAxiophot microscope using Nomarski optics or with a MRC1024 confocal microscope.

\section{Acknowledgments}

We thank X. Franch-Marro, S. González-Crespo, G. Jiménez, and M. Llimargas for discussions and critical comments on the manuscript. We also thank N. Martín for his technical assistance. M.B. was supported by an ARC and an EMBO fellowship. M.B. thanks D. Cribbs for accomodating her in the Centre de Biologie du Développement while revising the manuscript. This work was supported by a Royal Society University Research Fellowship and Wellcome Trust project grant 054613 to M.D.M.B, by a Senior Fellowship from the Wellcome Trust to N.H.B., and by grants from the CIRIT of the Generalitat de Catalunya and the Ministerio de Educación y Cultura de España to J.C.

The publication costs of this article were defrayed in part by payment of page charges. This article must therefore be hereby marked "advertisement" in accordance with 18 USC section 1734 solely to indicate this fact.

\section{References}

Brand, A.H. and Perrimon, N. 1993. Targeted gene expression as a means of altering cell fates and generating dominant phenotypes. Development 118: 401-415.

Brower, D.L., Wilcox, M., Piovant, M., Smith, R.J., and Reger, L.A. 1984. Related cell-surface antigens expressed with positional specificity in Drosophila imaginal discs. Proc. Nat1. Acad. Sci. 81: 7485-7489.

Brower, D.L., Bunch, T.A., Mukai, L., Adamson, T.E., Wehrli, M., Lam, S., Friedlander, E., Roote, C.E., and Zusman, S. 1995. Nonequivalent requirements for PS1 and PS2 integrin at cell attachments in Drosophila: Genetic analysis of the $\alpha$ PS1 integrin subunit. Development 121: 1311-1320.

Brown, N.H., Gregory, S.L., and Martin-Bermudo, M.D. 2000. Integrins as mediators of morphogenesis in Drosophila. Dev. Biol. 223: 1-16.

Campos-Ortega, J.A. and Hartenstein, V. 1985. The embryonic development of Drosophila melanogaster. Springer Verlag, Berlin/New York.

Chen, C.-K., Kühnlein, R.P., Eulenberg, K.G., Vincent, S., Affolter, M., and Schuh, R. 1998. The transcription factors KNIRPS and KNIRPS RELATED controll cell migration and branch morphogenesis during Drosophila tracheal development. Development 125: 4959-4968.

Cheresh, D.A. and Mecham, R.P. 1994. Integrins: Molecular and biological responses to the extracellular matrix. Academic Press, London, UK.

Chihara, T. and Hayashi, S. 2000. Control of tracheal tubulogenesis by Wingless signaling. Development 127: 4433-4442.

Chou, T.B. and Perrimon, N. 1992. Use of a yeast site-specific recombinase to produce female germline chimeras in Drosophila. Genetics 131: 643-653.

Franch-Marro, X. and Casanova, J. 2000. The alternative migratory pathways of the Drosophila tracheal cells are associated with distinct subsets of mesodermal cells. Dev. Biol. 270: 80-90.

Hidalgo, A., Urban, J., and Brand, A.H. 1995. Targeted ablation 
of glia disrupts axon tract formation in the Drosophila CNS. Development 121: 3703-3712.

Hogan, B.L.M. and Yingling, J.M. 1998. Epithelial/mesenchymal interactions and branching morphogenesis of the lung. Curr. Opin. Genet. Dev. 8: 481-486.

Hynes, R.O. 1992. Integrins: Versatility, modulation, and signaling in cell adhesion. Cell 69: 11-25.

Kühnlein, R.P. and Schuh, R. 1996. Dual function of the regionspecific homeotic gene spalt during Drosophila tracheal system development. Development 122: 2215-2223.

Leptin, M., Bogaert, T., Lehmann, R., and Wilcox, M. 1989. The function of PS integrins during Drosophila embryogenesis. Cell 56: 401-408.

Llimargas, M. 2000. Wingless and its signalling pathway have common and separable functions during tracheal development. Development 127: 4407-4417.

Llimargas, M. and Casanova, J. 1997. ventral veinless, a POU domain transcription factor, regulates different transduction pathways required for tracheal branching in Drosophila. Development 124: 3273-3281.

Manning, G. and Krasnow, M.A. 1993. Development of the Drosophila tracheal system. In The development of Drosophila melanogaster. (ed. M. Bate and A. Martínez-Arias). Vol. I, pp. 609-685, Cold Spring Harbor Laboratory Press, Cold Spring Harbor, NY.

Manoukian, A. and Krause, H.M. 1992. Concentration-dependent activities of the even-skipped protein in Drosophila embryos. Genes \& Dev. 6: 1740-1751.

Martin-Bermudo, M.D., Dunin-Borkowski, O.M., and Brown, N.H. 1997. Specificity of PS integrin function during embryogenesis resides in the $\alpha$ subunit extracellular domain. EMBO J. 16: 4184-4193.

Martin-Bermudo, M.D., Alvarez-Garcia, I., and Brown, N.H. 1999. Migration of the Drosophila primordial midgut cells requires coordination of diverse PS integrin functions. Development 126: 5161-5169.

Metzger, R.J. and Krasnow, M.A. 1999. Genetic control of branching morphogenesis. Science 284: 1635-1639.

Pai, L.M., Orsulic, S., Bejsovec, A., and Peifer, M. 1997. Negative regulation of Armadillo, a Wingless effector in Drosophila. Development 124: 2255-2266.

Perrimon, N., Noll, E., McCall, K., and Brand, A. 1991. Generating lineage-specific markers to study Drosophila development. Dev. Genet. 12: 238-252.

Roote, C.E. and Zusman, S. 1995. Functions for PS integrins in tissue adhesion, migration and shape changes during early embryonic development in Drosophila. Dev. Biol. 169: 322336.

Rothe, M., Nauber, U., and Jäckle, H. 1989. Three hormone receptor-like Drosophila genes encode an identical DNAbinding finger. EMBO J.. 8: 3087-3094.

Samakovlis, C., Hacohen, N., Manning, G., Sutherland, D.C., Guillemin, K., and Krasnow, M.A. 1996. Development of the Drosophila tracheal system occurs by a series of morphologically distinct but genetically coupled branching events. Development 122: 1395-1407.

Shiga, Y., Tanaka-Matakatsu, M., and Hayashi, S. 1996. A nuclear GFP/B-galactosidase fusion protein as a marker for morphogenesis in living Drosophila. Dev. Growth Differ. 38: 99-106.

Sutherland, D., Samakovlis, C., and Krasnow, M.A. 1996. branchless encodes a Drosophila FGF homolog that controls tracheal cell migration and the pattern of branching. Cell 87: 1091-1101.

Vincent, S., Ruberte, E., Grieder, N.C., Chen, C., Haerry, T., Schuh, R., and Affolter, M. 1997. DPP controls tracheal cell migration along the dorsoventral body axis of the Drosophila embryo. Development 124: 2741-2750.

Wappner, P., Gabay, L., and Shilo, B-Z. 1997. Interactions between the EGF receptor and DPP pathways establish distinct cell fates in the tracheal placodes. Development 124: 47074716.

Wilcox, M., Brown, N., Piovant, M., Smith, R.J., and White, R.A. 1984. The Drosophila position-specific antigens are a family of cell surface glycoprotein complexes. EMBO J. 3: 23072313.

Wu, J.E. and Santoro, S.A. 1996. Differential expression of integrin alpha subunits supports distinct roles during lung branching morphogenesis. Dev. Dyn. 206: 169-181. 


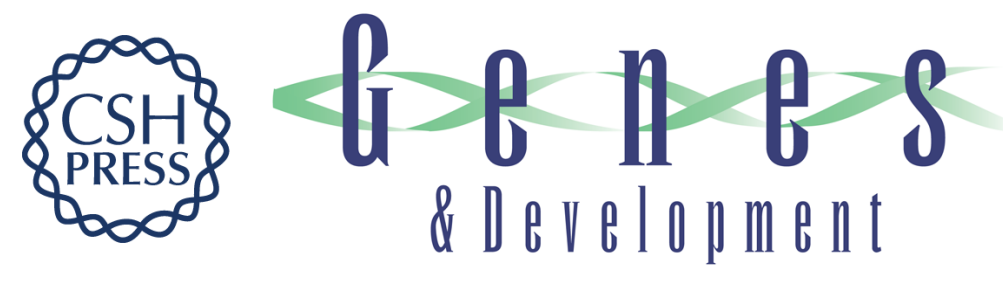

\section{Specific tracheal migration is mediated by complementary expression of cell surface proteins}

Muriel Boube, Maria D. Martin-Bermudo, Nicholas H. Brown, et al.

Genes Dev. 2001, 15:

Access the most recent version at doi:10.1101/gad.195501

References

This article cites 28 articles, 18 of which can be accessed free at: http://genesdev.cshlp.org/content/15/12/1554.full.html\#ref-list-1

License

Email Alerting

Receive free email alerts when new articles cite this article - sign up in the box at the top Service right corner of the article or click here.

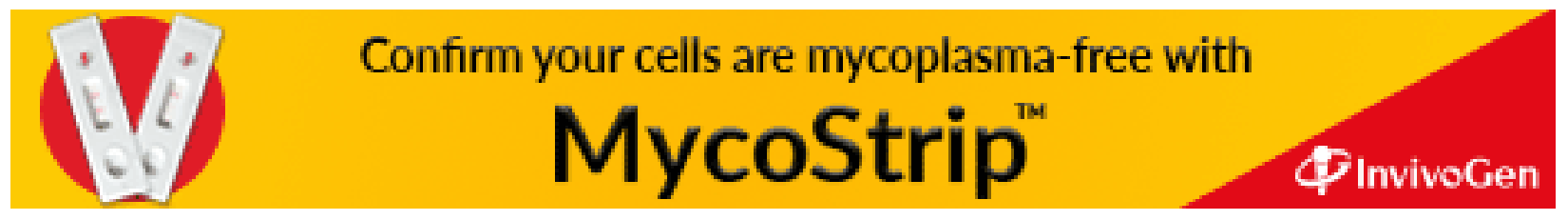

